\title{
Migration of $\mathrm{CO}_{2}$ in Porous Media Filled with water."
}

\author{
Tetsuya SUEKANE $^{* *}$ Toshihiro ISHII ${ }^{* *}$ \\ Shoji TSUSHIMA ${ }^{* *}$ and Shuichiro HIRAI** \\ ${ }^{* *}$ Research Center for Carbon Recycling and Energy, Tokyo Institute of Technology, \\ 2-12-1 Ohokayama, Meguro, Tokyo, 152-8552 JAPAN \\ E-mail: tsuekane@mep.titech.ac.jp
}

\begin{abstract}
This paper describes experimental research on two-phase flow of supercritical carbon dioxide $\left(\mathrm{CO}_{2}\right)$ and water in porous media under sequestration conditions. We used a magnetic resonance imaging technique to directly visualize the distribution of supercritical $\mathrm{CO}_{2}$ injected into porous media containing water. In-situ water saturation distributions in a Berea sandstone core and a packed bed of glass beads were successfully visualized at conditions that simulate those at $700-$ $1000-\mathrm{m}$ depths in an aquifer. By applying a coreflood interpretation method to our saturation data, we could describe the local Darcy phase velocities and capillary dispersion rate as a function of saturation. Next, the effect of buoyancy on the displacement process and gravity-dominated pure counter-current flow is discussed by comparing the data obtained at two conditions that model the aquifers at depths of $700 \mathrm{~m}$ and $1000 \mathrm{~m}$. We found that buoyancy largely controls the pure counter-current flow in permeable porous media for conditions at 700 and $1000-\mathrm{m}$ depths.
\end{abstract}

Key words: Porous Media, Magnetic Resonance Imaging, Carbon Dioxide, Geological Storage

\section{Introduction}

Carbon dioxide is considered to be the greenhouse gas that most contributes to global warming on earth. In response to this problem, several methods have been proposed to remove $\mathrm{CO}_{2}$ from the atmosphere. Sequestration of $\mathrm{CO}_{2}$ in geological formations such as aquifers is one such method. In aquifers, the critical condition of $\mathrm{CO}_{2}$ is reached at a depth of about $700 \mathrm{~m}$. At depths of 1000 to $3000 \mathrm{~m}$, the viscosity of supercritical $\mathrm{CO}_{2}$ is 0.05 to 0.1 times that of water and the density of $\mathrm{CO}_{2}$ is 0.6 times that of water. Therefore, buoyancy tends to bring the sequestered $\mathrm{CO}_{2}$ back to the surface unless there is a confining impermeable stratum (caprock). A potential problem with geological sequestration is the leakage of $\mathrm{CO}_{2}$ from reservoirs.

Detail of storage process is considered as follows [1]. In saline formations, buoyancy forces drive the $\mathrm{CO}_{2}$ upwards towards the highest point of the caprock as shown in Fig. 1. Physical trapping below the sealing caprock is the principle mechanism to store $\mathrm{CO}_{2}$. As $\mathrm{CO}_{2}$ migrates away from the injection well some of it will be retained in the pore space as non-wetting phase by capillary pressure. This trapping mechanism is often referred as residual gas trapping. Simultaneously, $\mathrm{CO}_{2}$ will dissolve in the pore water. Some researchers have suggested that convection will be created by the density difference between carbonate water and pure water, but it takes more than hundreds years to dissolve a 


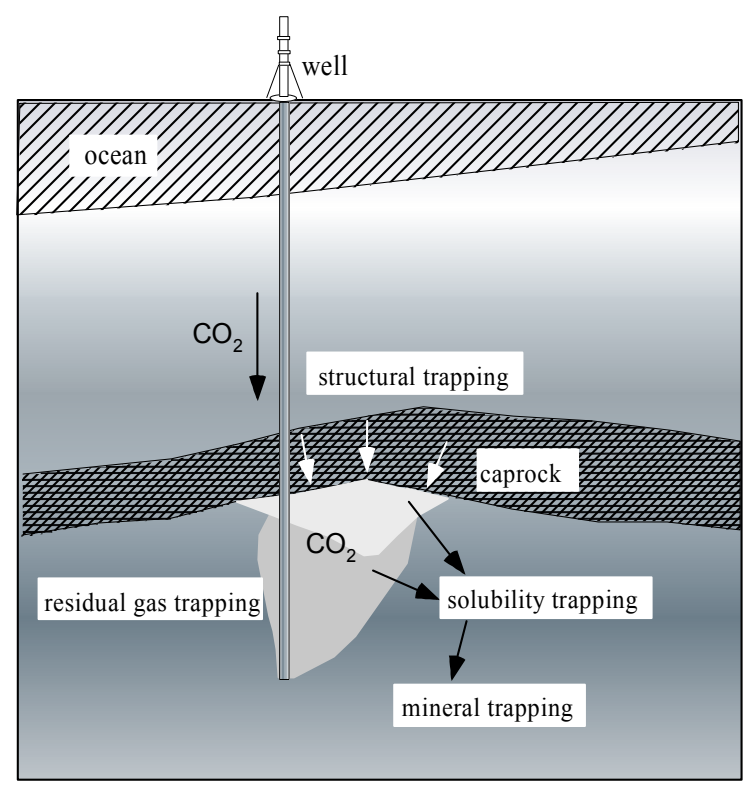

Fig. 1 Trapping mechanism.

large amount of injected $\mathrm{CO}_{2}$ into water [2-4]. Mineral trapping is expected to be slow but, over long time scales, may trap a significant fraction of the $\mathrm{CO}_{2}[3,5-7]$.

Qualitatively, the long-term fate of injected $\mathrm{CO}_{2}$ into geological formations is expected as mentioned above. However, it is still very difficult to predict when $\mathrm{CO}_{2}$ will be stabilized by dissolution into water or how much amount of $\mathrm{CO}_{2}$ will be stabilized by each trapping mechanism at a certain time point, because of the lack of information about the property of migration and dissolution of $\mathrm{CO}_{2}$. Since the dissolution of $\mathrm{CO}_{2}$ into water is governed by natural convection due to the difference in density [2-4], the dissolution process is greatly affected by the migration property of $\mathrm{CO}_{2}$. From the view point of risk assessment, the estimation of leakage rate through the fault of the caprock is required. As discussed later, the migration of $\mathrm{CO}_{2}$ is very complicated, because it is affected by viscosity, buoyancy, and capillary pressure. Moreover, the viscosity, density, and interfacial tension change sharply around its critical point, which corresponds to the depths in the range between $700 \mathrm{~m}$ and $1000 \mathrm{~m}$. Therefore, understandings of the migration of $\mathrm{CO}_{2}$ in these conditions are quite important for prediction of stored $\mathrm{CO}_{2}$ in geological formations.

This paper describes experimental research on two-phase flow of supercritical $\mathrm{CO}_{2}$ and water in porous media at sequestration conditions. We used a magnetic resonance imaging (MRI) technique to directly visualize the distribution of supercritical $\mathrm{CO}_{2}$ in a Berea sandstone core and a packed bed of glass beads containing water. First we discuss the characteristics of the displacement process of water by supercritical $\mathrm{CO}_{2}$ in Berea sandstone. Recently, Goodfield et al. [8] proposed a new interpretation method that fully utilizes in-situ saturation data obtained from methods such as X-ray computer tomography scanning and MRI. We apply MRI to evaluate the properties of two-phase flow such as local Darcy phase velocities and the capillary dispersion rate for a range of saturations. Next, we discuss the effect of buoyancy on the displacement process and gravity-dominated pure counter-current flow by comparing the data obtained at two conditions that model aquifers at depths of 700 and $1000 \mathrm{~m}$. We used a packed bed of glass beads, where the effect of buoyancy is significant due to its high permeability and porosity.

\section{Experimental Apparatus and Measurement Techniques}

We performed experiments on a consolidated and layered Berea sandstone sample and a packed bed of glass beads with a $70-\mu \mathrm{m}$ average diameter. The experimental setup for both 
media was practically the same; therefore, we describe the details only for the sandstone case.

\section{Experimental apparatus}

A schematic diagram of the experimental setup for sandstone cores is shown in Fig. 2 (a). A high pressure vessel containing a sandstone core was inserted vertically into the MRI system. The experimental setup consisted of two high pressure circuits, namely, the process line and the fluorinert circulation line. The process line feeds water or supercritical $\mathrm{CO}_{2}$ to the sandstone core. Pure water or liquid $\mathrm{CO}_{2}$ was forced into the process line by a water pump (Nihon Seimitsu NP-KX-500-40, 0.04-40 ml/min) or $\mathrm{CO}_{2}$ pump (Nihon Bunko $\mathrm{SCF}-\mathrm{Get} 0.2-10 \mathrm{ml} / \mathrm{min}$ ), respectively. The flow rate and back pressure were controlled by these pumps and a pressure regulator (Nihon Bunko SCF-Bpg 1.0-50 MPa). The pressure drop through the sandstone core was measured using low differential pressure transmitters (GE Druck LPX9318, 0-10 kPa, 0-1 MPa). The fluorinert circulation line provides the sandstone core with an overburden pressure that was about 1-MPa higher than the injection pressure of $\mathrm{CO}_{2}$. Usually, researchers use water for the overburden pressure medium; however, the protons in water molecules emit MR signals of high intensity that reduce $\mathrm{S} / \mathrm{N}$ ratio of signals from the sandstone core. Therefore, we used fluorinert, which has no single proton and is chemically inert. Accumulators were connected in these two lines to reduce pressure pulsations and flow rate. Except for the two high pressure lines, there was a normal pressure line of water to control the temperature of the sandstone core and injecting fluids constant and uniform.

The pressure cell used in the present MRI measurements is shown in Fig. 2 (b). As mentioned briefly below, an MRI technique requires a uniform and high magnetic field applied on visualized materials. For this, the pressure vessel was constructed of a polyamide-imide pipe with $26-\mathrm{mm}$ inner diameter and $50-\mathrm{mm}$ outer diameter. This material was selected because it is nonmagnetic and hence should not introduce any inhomogeneities to the magnetic field and should not interfere with the RF signals used in the experiments. A

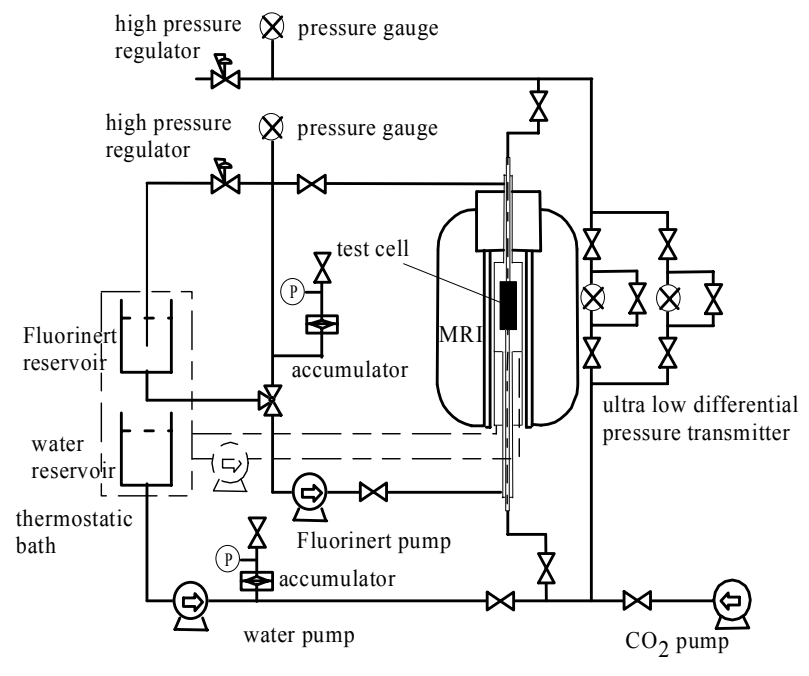

(a)

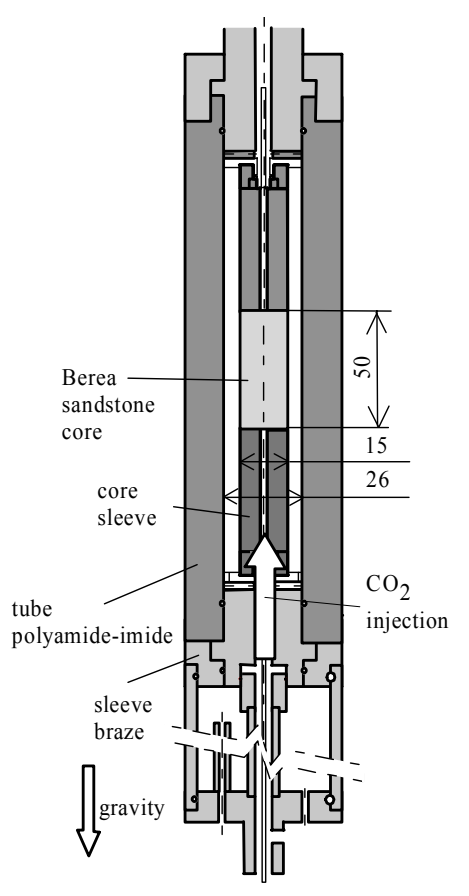

(b)

Fig. 2 The experimental setup (a) and cross-sectional view of the core holder used in the experiments (b). All dimensions are in millimeters. 
Berea sandstone core $15-\mathrm{mm}$ in diameter and 50-mm long was used in the experiments. The sandstone layers were oriented such that the normal to each layer surface was parallel to the cylinder axis. The sandstone core was coated with sleeves made from Teflon heat shrink tubing to insulate the hydraulic connection between fluid in the sandstone and the fluorinert. The sleeves were connected to coaxial pipes that conduct water and $\mathrm{CO}_{2}$ in the inner tube and fluorinert in the outer tube. Temperatures at the inlet and exit of the sandstone core were measured using thermocouples inserted into the coaxial pipes.

For the packed bed of glass beads, we packed glass beads with a $70-\mu \mathrm{m}$ average diameter into a polyamide-imide pipe with inner diameter of $26 \mathrm{~mm}$ and about $50-\mathrm{mm}$ long. More details of the high-pressure vessel are in refs. [9-10]. To pack the glass beads uniformly, the high-pressure vessel was connected to a vacuum chamber and the packing was achieved under high-speed air flow. The Berea had a permeability of $23.9 \mathrm{mD}$ and an average porosity of $15.8 \%$. The packed bed of glass beads had a permeability of $284 \mathrm{mD}$, more than 10 times that of the sandstone, and a porosity of $38 \%$.

\section{MRI measurement techniques}

We used the MRI measurement technique to image the water distribution in axial and transaxial sections through Berea sandstone and the packed bed of glass beads. Noninvasive measurement of a water distribution is one of the advantages of the MRI technique. For example, Merrill and Jin [11] and Mansfield and Issa [12] measured the local velocity of water injected into Casper and Bentheimer sandstones, respectively. Authors used our MRI equipment to measure the local velocity distributions in porous media [9-10, 13]. The details of the MRI techniques have been published elsewhere [14, 15]. We will, therefore, restrict our discussion to the general principle of MRI measurements.

Nuclei with non-zero spin angular momenta have an intrinsic magnetic dipole moment. In a static magnetic field, $B_{0}$, each nuclear spin precesses with frequency, $\omega_{0}=\gamma B_{0}$, where $\omega_{0}$ is called the Larmor frequency and $\gamma$ is the gyromagnetic ratio, characteristic of the nuclei being studied. The Larmor frequency for protons at $7.05 \mathrm{~T}$, which is the strength of magnetic field used in our MRI system (Varian Unity Inova model 300/150 SWB), is about $300 \mathrm{MHz}$. By applying a radio frequency magnetic field at the Larmor frequency, the nuclear magnetic resonance signal due to precession is observed after the magnetization is rotated away from the static field. In MRI, spatial information is converted into the MR signals by applying a gradient magnetic field. Hence, the distribution of proton density can be imaged in any cross-section. In our experiments, the gradient coils provided a maximum gradient strength of $0.24 \mathrm{~T} / \mathrm{m}$.

In preliminary experiments, we confirmed that the MR signal intensity from any local position was proportional to the water content in the porous medium. This means that measured MR signal intensity reflects local water saturation, which is defined as the fraction of the void volume occupied by water [16], in the porous media. In the experiments with $\mathrm{CO}_{2}$ injection, we measured the initial MR signal intensity distributions in the porous medium filled with water. Then, we started to supply $\mathrm{CO}_{2}$ into the porous medium with time-series acquisition of MR images. The injected $\mathrm{CO}_{2}$ would replace some water in the porous medium thus decreasing the MR signal intensity. The local water saturation in the porous medium can be defined as the ratio of MR signal intensity with the $\mathrm{CO}_{2}$ gas to that without gas.

\section{Core analysis methods}

Darcy law for two phase flow [17] is expressed as:

$$
U_{\alpha}=-k \lambda_{\alpha}\left(\frac{\partial p_{\alpha}}{\partial z}+\rho_{\alpha} g\right)
$$




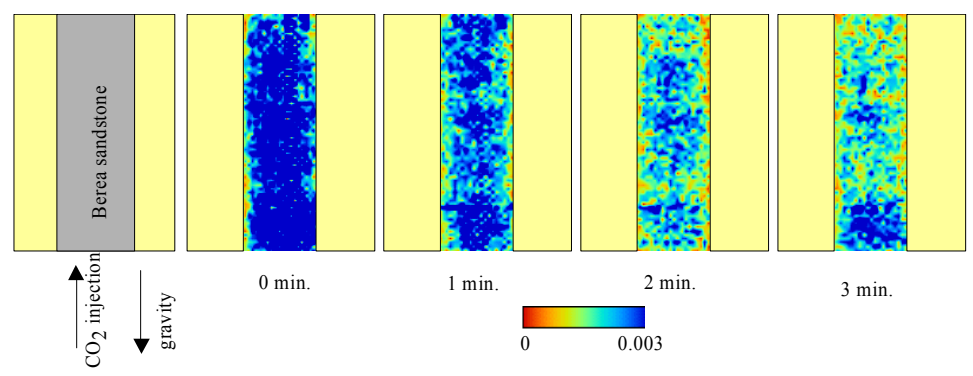

Fig. 3 Distribution of water in Berea sandstone core at $9.0 \mathrm{MPa}, 42{ }^{\circ} \mathrm{C}$ with $\mathrm{CO}_{2}$ injection rate of $3.0 \mathrm{ml} / \mathrm{min}$. Arbitrary unit.

where $\alpha$ denotes each phase of $\mathrm{CO}_{2}(g)$ and water $(w) ; U$, local Darcy velocity; $\lambda_{\alpha}=k_{r \alpha} / \mu_{\alpha}$, mobility; $k$, absolute permeability; $p$, pressure; $\rho$, density; g, gravitational acceleration; $k_{r}$, relative permeability; $\mu$, viscosity. The capillary pressure is defined by:

$$
p_{c w}\left(S_{w}\right)=p_{w}\left(S_{w}\right)-p_{g}\left(S_{w}\right)
$$

From equations (1) and (2), we can represent the local Darcy velocity of $\mathrm{CO}_{2}$ as [8]:

$$
U_{g}=U(t) \frac{\lambda_{g}}{\lambda_{w}+\lambda_{g}}\left[1+\frac{k g\left(\rho_{w}-\rho_{g}\right)}{U(t)} \lambda_{w}\right]-k \frac{\lambda_{g} \lambda_{w}}{\lambda_{g}+\lambda_{w}} \frac{d p_{c w}}{d S_{w}} \frac{\partial S_{w}}{\partial z}
$$

where $U$ is the total flow rate. The first and second terms in the bracket of first term of RHS denote the effects of viscosity and buoyancy, respectively, and the second term of RHS denotes the effect of capillary pressure on the local Darcy velocity of $\mathrm{CO}_{2}$. Goodfiled et al. [8] has shown that these three terms can be evaluated independently by imaging the distribution of each phase in the core at various injection flow rates. The relative permeability and the capillary dispersion rate, which are used for the reservoir simulations, can be evaluated without the additional measurement such as pressure drop across the core. However, in present study, due to the limitation of our pumping system, the distribution of each phase in the core has been imaged at single flow rate. Therefore, the local Darcy velocity and the capillary dispersion rate function will be evaluated.

\section{Experimental Results and Discussion}

\section{Characteristics of displacement process of water by supercritical $\mathrm{CO}_{2}$}

Distributions of the MR signal at various times, that is, water and $\mathrm{CO}_{2}$ in the Berea sandstone core are shown in Fig. 3. Supercritical $\mathrm{CO}_{2}$ was injected vertically upward into the sandstone core saturated with water at a pressure of $9.0 \mathrm{MPa}$ and a temperature of $42{ }^{\circ} \mathrm{C}$ characteristic of aquifers at a depth of about $900 \mathrm{~m}$. The flow rate of injected $\mathrm{CO}_{2}$ was 3.0 $\mathrm{ml} / \mathrm{min}$ in its liquid phase. At this flow rate, the capillary number is in the order of $10^{-6}$, which is low enough to prevent the effect of phase trapping. The leftmost image shows a water saturated core before the injection of $\mathrm{CO}_{2}$. The height of the image is about $50 \mathrm{~mm}$, and the top and bottom of the image correspond to the exit and inlet of the cylindrical core. The ratio of the MR signal of each pixel after injection of $\mathrm{CO}_{2}$ to this initial image denotes water saturation. The remaining three images in Fig. 3 show the distribution of the MR signal after the injection of $\mathrm{CO}_{2}$. The moment when the intensity of MR signal was first reduced by intrusion of $\mathrm{CO}_{2}$ is taken to be $t=0$. The replacement of water by injected $\mathrm{CO}_{2}$ caused the MR signal intensity to decrease with time as shown in the figure. The experiment also demonstrates that the two-phase flow in sandstone at the condition of geological sequestration could be visualized by the MRI technique. Even after $\mathrm{CO}_{2}$ breaks through the sandstone core $(t=3 \mathrm{~min}$.), water concentration fluctuates along the core. This fluctuation of water concentration results from the distribution in porosity along the axial direction due 


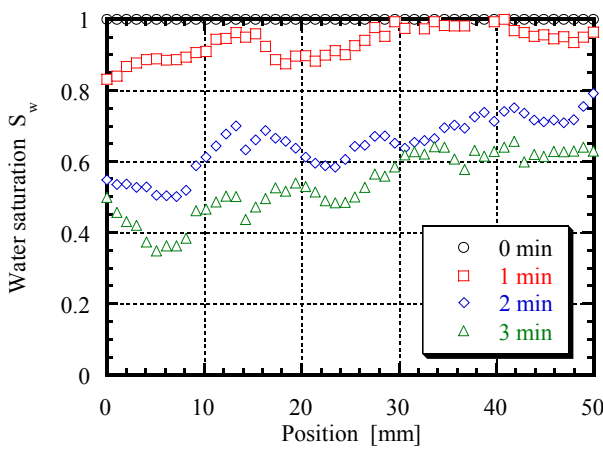

Fig. 4 Time evolution of water saturation at $9.0 \mathrm{MPa}, 42^{\circ} \mathrm{C}$ with $\mathrm{CO}_{2}$ injection rate of $3.0 \mathrm{ml} / \mathrm{min}$.

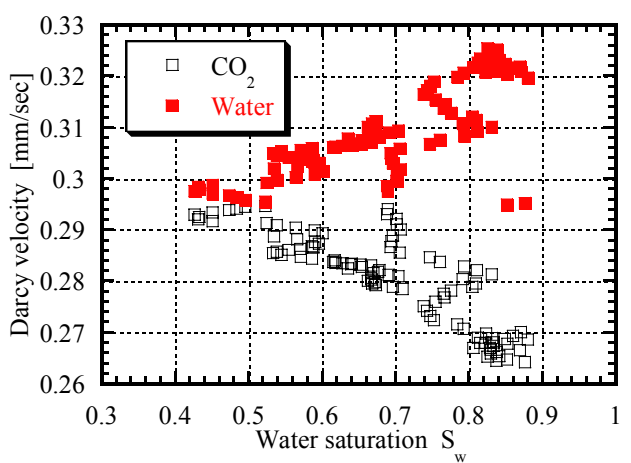

Fig. 5 Local Darcy phase velocities as a function of water saturation at $9.0 \mathrm{MPa}$, $42^{\circ} \mathrm{C}$.

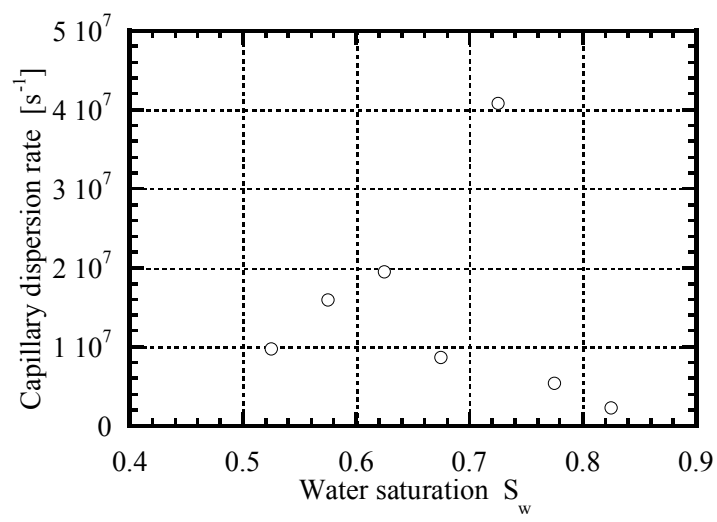

Fig. 6 Capillary dispersion rate of a

Berea sandstone core at $9.0 \mathrm{MPa}, 42^{\circ} \mathrm{C}$.

to the sedimentary structure of sandstone.

The time evolution of water saturation along the core is shown in Fig. 4. The water saturation was calculated in the following manner. First, the water saturation in each pixel was calculated as the ratio of the MR signal with the $\mathrm{CO}_{2}$ gas to that without gas $(t=0)$. Next, the water saturation was averaged in a lateral direction within the core, that is, for a given position $z$ along the flow direction from the inlet of the core $(z=0)$. With the injection of $\mathrm{CO}_{2}$, the water saturation decreases gradually from the inlet of the sandstone core. The water saturation decreases down to about $0.4-0.6$, where water tends to be immobilize due to low relative permeability after $\mathrm{CO}_{2}$ breaks through the core. Judging form distributions of saturation in the lateral direction, saturation has the error of about $10 \%$.

The coreflood interpretation method proposed by Goodfield et al. [8] was applied to our data to determine the local Darcy phase velocities in the sandstone core. The phase volumes per unit cross-sectional area between the core inlet $(z=0)$ and position $z>0$ are given by

$$
V_{g}=\int_{0}^{z} \phi(\varsigma) S_{g}(\varsigma, t) d \varsigma
$$

where $\phi(z)$ is the porosity at position $z$ and $S_{g}(z, t)$ is the $\mathrm{CO}_{2}$ saturation at position $z$ and time $t$. Using a material balance approach, the local $\mathrm{CO}_{2}$ Darcy phase velocity can then be expressed in the following form:

$$
U_{g}(z, t)=U(t) F_{g}^{i n j}(t)-\frac{\partial V_{g}(z, t)}{\partial t}
$$


where $U_{g}(z, t)$ is the local Darcy phase velocity of $\mathrm{CO}_{2}, U(t)$ is the total Darcy velocity, and $F_{g}^{i n j}(t)$ is the fractional flow of $\mathrm{CO}_{2}$ injected at time $t$.

The local Darcy phase velocities of $\mathrm{CO}_{2}$ and water obtained with this method are shown in Fig. 5 for various levels of water saturation. The phase velocity of $\mathrm{CO}_{2}$ decreases and that of water increases with an increasing water saturation.

Next, the capillary dispersion rate function [8]

$$
d_{c p w}\left(S_{w}\right)=-\frac{\lambda_{g} \lambda_{w}}{\lambda_{g}+\lambda_{w}} \frac{d p_{c w}}{d S_{w}}
$$

in equation (3) obtained from the in-situ saturation data is shown in Fig. 6 for a sandstone core. Except for one point around the water saturation of 0.73 , the obtained capillary dispersion rate function shows smooth distribution for the water saturation. It is worth noting that this capillary dispersion rate can be estimated from only in-situ phase distribution data and the injection flow rate. In other words, without the measurement of capillary pressure function $p_{c w}$, the capillary dispersion rate function can be evaluated. The water saturation measured by MRI has an error of about $10 \%$. However, as demonstrated by Goodfield et al. [8], the capillary dispersion rate function is estimated by a nonlinear way, therefore, accuracy changes over the saturations.

In a future study, we plan to measure the in-situ saturation profiles for various flow rates of $\mathrm{CO}_{2}$ injection so that we can quantitatively estimate the capillary pressure function and the relative permeabilities of $\mathrm{CO}_{2}$ and water.

\section{Effect of buoyancy}

We used a packed bed of glass beads to investigate the effect of buoyancy in two-phase flow of supercritical $\mathrm{CO}_{2}$ and water, because the effect of buoyancy is significant due to high permeability and porosity of the packed bed. Before discussing the effect of buoyancy, the characteristics of displacement process for the packed bed is briefly introduced. The procedure used was the same as that with the Berea sandstone; supercritical $\mathrm{CO}_{2}$ was injected vertically upward into a packed bed of glass beads saturated with water. The experiment was carried out at a pressure of $8.0 \mathrm{MPa}$ and a temperature of $40{ }^{\circ} \mathrm{C}$, which are conditions characteristic of aquifers at a depth of about $800 \mathrm{~m}$. The flow rate of the injected $\mathrm{CO}_{2}$ was $4.0 \mathrm{ml} / \mathrm{min}$.

The packed bed of glass beads produced clearer images than the sandstone core because

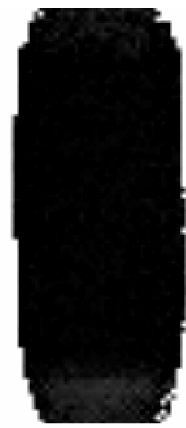

(a) $0 \mathrm{~s}$

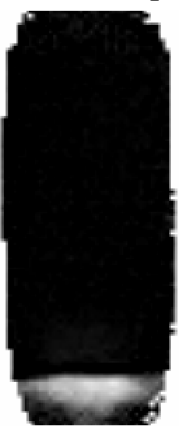

(b) $30 \mathrm{~s}$

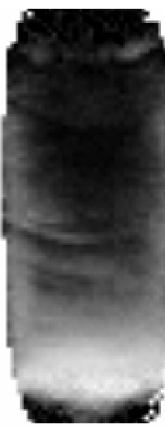

(c) $60 \mathrm{~s}$

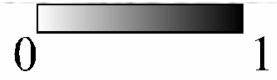

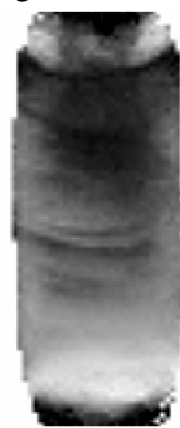

(d) $90 \mathrm{~s}$

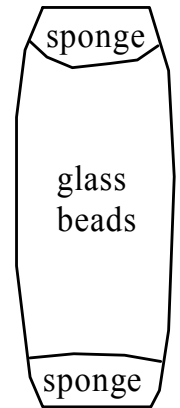

1

Fig. 7 Water saturation at various times in the vertical plane of a packed bed of glass beads. The water saturation was averaged over a depth of $2 \mathrm{~mm}$ in a lengthwise section through the center of the cylinder. Conditions were $40{ }^{\circ} \mathrm{C}$ and $8.0 \mathrm{MPa}$. 
the glass beads have a homogeneous porous structure with greater porosity. Figure 7 shows the distribution of water saturation in the packed bed. The images show several things. First, the sequence a-d shows the light region appearing at the bottom and moving up. This shows the general motion of $\mathrm{CO}_{2}$ from the bottom towards the top, which is the imposed flow direction. Second, the $\mathrm{CO}_{2}$ appears to move faster along the sides and slowest in the middle. This may be a result of the packing; the glass beads tend to be packed such that there is a higher porosity near the tube wall. The result is denser packing and thus smaller porosity in the middle of the sample. Third, at 30 seconds and later (images b-d), the interface between

Table 1 Experimental conditions.

\begin{tabular}{|l|c|c|c|l|c|}
\hline & $\begin{array}{l}\mathrm{T} \\
{\left[{ }^{\circ} \mathrm{C}\right]}\end{array}$ & $\begin{array}{l}\mathrm{p} \\
{[\mathrm{MPa}]}\end{array}$ & $\begin{array}{l}\rho_{\mathrm{g}} \\
{\left[\mathrm{kg} / \mathrm{m}^{3}\right]}\end{array}$ & $\begin{array}{l}\mu_{\mathrm{g}} \\
{[\mathrm{Pa} \mathrm{s}]}\end{array}$ & $\begin{array}{l}\text { Equivalent } \\
\text { depth }[\mathrm{m}]\end{array}$ \\
\hline A & 50 & 10 & 353 & $1.95 \times 10^{-5}$ & 1000 \\
\hline B & 32 & 7.4 & 261 & $1.77 \times 10^{-5}$ & approx. 700 \\
\hline
\end{tabular}

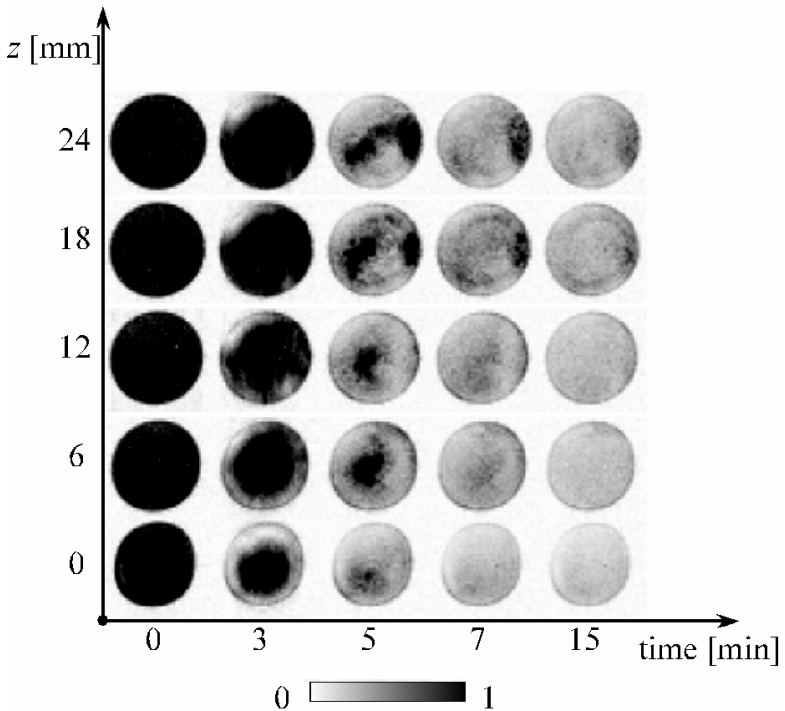

Fig. 8 Distribution of water saturation at five positions along the length of core under condition $\mathrm{B}\left(32^{\circ} \mathrm{C}, 7.4 \mathrm{MPa}\right)$.

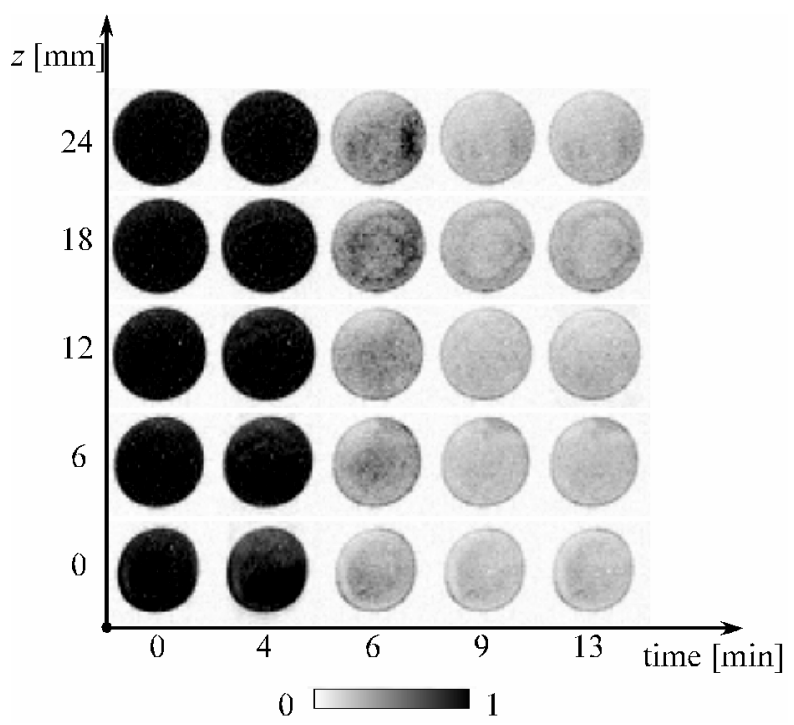

Fig. 9 Distribution of water saturation at five positions along the length of core under condition $\mathrm{A}\left(50^{\circ} \mathrm{C}, 10 \mathrm{MPa}\right)$. 
the packed bed and sponge rubber gasket (that holds the bed) is clearly imaged. At 90 seconds, the top gasket also shows in the image. Finally, we note that the method introduces some artifacts; the cylinder diameter appears slightly narrower at the top and bottom, and also the curved, dark bands in images $\mathrm{c}$ and $\mathrm{d}$.

We investigated the effect of buoyancy on two-phase flow in a packed bed of glass beads. Supercritical $\mathrm{CO}_{2}$ was injected into the packed bed of glass beads for two conditions with different densities (Table 1). Condition A approximates aquifers at a depth of about $1000 \mathrm{~m}$. In contrast, condition $\mathrm{B}$ approximates aquifers at a depth of about $700 \mathrm{~m}$ and $\mathrm{CO}_{2}$ is significantly less dense than $\mathrm{CO}_{2}$ at condition $\mathrm{A}$. The properties at both conditions are listed in Table 1.

The distributions of water saturation in horizontal cross-sections along the flow direction under the conditions A and B are shown in Figs. 8 and 9, respectively. As found for the vertical cross-sections, the lateral cross-sections show that $\mathrm{CO}_{2}$ tends to penetrate into the packed bed through the more permeable regions near the outer edge. Under the higher-pressure of condition $\mathrm{A}$, the distribution of water saturation reaches its steady state in 9 minutes. After that, water saturation is uniform in each cross-section. However, a water saturation of about $30 \%$ remains in the packed bed due to capillary pressure. On the other hand, under the lower-pressure of condition $\mathrm{B}$, the dark regions in the cross-sections at 18 and $24 \mathrm{~mm}$ indicate that some water remains in the pores. For example, more detailed analyses of the cross-sections indicate that roughly $80 \%$ of the pores are filled with water on one side of the cross-section. This is because the work done by the pressure gradient, which forces the water out, decreases after some $\mathrm{CO}_{2}$ reaches the exit of the packed bed. Some $\mathrm{CO}_{2}$ first exits the bed in 5 minutes. Therefore, the top of the column is still losing water five minutes after the bottom has reached the minimum saturation level of about 0.25.

At condition $\mathrm{B}$, the density and viscosity of supercritical $\mathrm{CO}_{2}$ are 0.74 and 0.9 times lower than those for condition $\mathrm{A}$. In other words, the $\mathrm{CO}_{2}$ is more buoyant and slightly less viscous under condition $\mathrm{B}$. Therefore, even for the injection process, $\mathrm{CO}_{2}$ spreads through more permeable regions due to buoyancy. After the $\mathrm{CO}_{2}$ paths reach the exit of the packed bed, the reduction of the pressure gradient along the bed results in a lower water sweep by the $\mathrm{CO}_{2}$. Under condition $\mathrm{A}$, the displacement of water by $\mathrm{CO}_{2}$ shows a more piston-like behavior, but the effect of buoyancy is still noticeable.

After $\mathrm{CO}_{2}$ injection stopped, $\mathrm{CO}_{2}$ in the packed bed moved upward due to buoyancy and a pure counter-current flow was established. Figure 10 shows the time evolution of water saturation in the cross-section at $24 \mathrm{~mm}$. We stopped the $\mathrm{CO}_{2}$ injection at 11 minutes for condition $\mathrm{A}$, and the water saturation did not reach $70 \%$ even after 30 minutes. However,

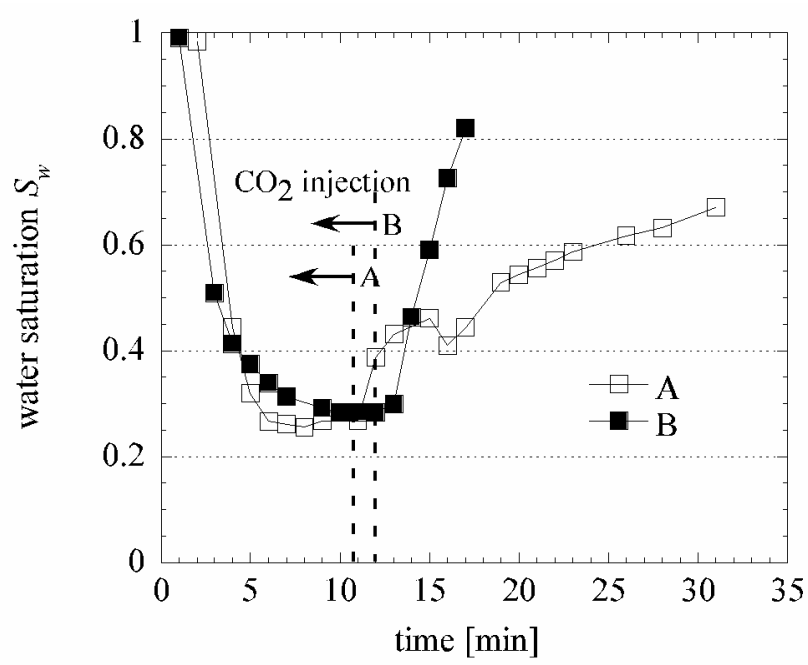

Fig. 10 Water saturation 24-mm from the inlet during and after the $\mathrm{CO}_{2}$ injection. 
with the more buoyant condition of $\mathrm{B}$, the water saturation was $80 \%$ only 4 minutes after the $\mathrm{CO}_{2}$ was shut off at 13 minutes. This indicates that the supercritical $\mathrm{CO}_{2}$ moves upward more rapidly due to buoyancy.

Even after much longer period, the water saturation will not recover $100 \%$, because some of $\mathrm{CO}_{2}$ will be trapped as non-wetting phase in the porous media by capillary force. This trap mechanism is often referred to as the residual gas trapping, which is one of the most important trapping in geological storage. For the prediction of long-term fate of $\mathrm{CO}_{2}$, understandings of trapping mechanism will be very important. The amount of trapped $\mathrm{CO}_{2}$ will depend on the temperature and pressure, because around the critical point of $\mathrm{CO}_{2}$ the interfacial tension, viscosity, and density change sharply.

\section{Conclusions}

This paper describes experimental research on two-phase flow of supercritical $\mathrm{CO}_{2}$ and water in porous media at sequestration conditions. We used the MRI technique to directly visualize the distribution of supercritical $\mathrm{CO}_{2}$ injected into a Berea sandstone core and a packed bed of glass beads containing water. The conditions were chosen to simulate temperature and pressure conditions typical of aquifers at depths of about 700- and 1000-m below ground - conditions under which $\mathrm{CO}_{2}$ may be injected in the future to sequester $\mathrm{CO}_{2}$ captured from anthropogenic sources.

We used the coreflood interpretation method to determine the local Darcy phase velocities and capillary dispersion rate for a range of water saturations. We successfully demonstrated that the properties of two-phase flow such as Darcy phase velocities and the capillary dispersion rate function can be estimated quantitatively from the in-situ saturation profiles obtained by MRI.

After the $\mathrm{CO}_{2}$ displaced much of the water, some water remained in the porous media due to capillary pressure. Since $\mathrm{CO}_{2}$ is more buoyant and less viscous near its critical point, the $\mathrm{CO}_{2}$ tended to penetrate through more permeable regions. Under the condition that approximates aquifers at the depth of $1000 \mathrm{~m}$, the displacement of water by $\mathrm{CO}_{2}$ shows more piston-like behavior, but the effect of buoyancy was still notable. After the injection of $\mathrm{CO}_{2}$ stopped, the migration of supercritical $\mathrm{CO}_{2}$ due to buoyancy was observed.

\section{References}

(1) Benson, S. M., Carbon Dioxide Capture for Storage in Deep Geologic Formation, Vol.2, (2005) 665-672.

(2) Gale, J., Proc. 6th Intern. Conf. on Greenhouse Gas Control Technologies, 1, (2002), 207.

(3) Pruess, K., and Xu, T., Apps, J., and Garcia, J., SPE J., 49, (2003).

(4) Ennis-King, J. and Paterson, L., Proc. 6th Intern. Conf. on Greenhouse Gas Control Technologies, (2002), 507.

(5) Gerdemann, S. J. , Dahlin, D. C., and O'Connor, W. K., Proc. 6th Intern. Conf. on Greenhouse Gas Control Technologies, 1, (2002), 677.

(6) Czernichowski-Lauriol, I., Rochelle, C. A., Brosse, E., Springer, N., Bateman, K., Kervevan, C., Pearce, J. M., Sanjuan, B., and Serra, H., Proc. 6th Intern. Conf. on Greenhouse Gas Control Technologies, 2, (2002), 1617.

(7) Obdam, A., van der Meer, L., May, F., Kervevan, C., Bech, N., and Wildenborg, A., Proc. 6th Intern. Conf. on Greenhouse Gas Control Technologies, 1, (2002), 339.

(8) Goodfield, G., S. G. Goodyear, and P. H. Townsley., New Coreflood Interpretation Method for Relative Permeabilities Based on Direct Processing In-Situ Saturation Data, the 2001 SPE Annual Technical Conference and Exhibition, SPE 71490 (2001)

(9) Suekane, T., Soukawa, S., Iwatani, S., Tsushima, S., and Hirai, S., Addendum to the Proc. of 6th Intern. Conf. on Greenhouse Gas Control Technologies, (2003) 5-10. 
(10) Suekane, T., Soukawa, S., Iwatani, S., Tsushima, S., and Hirai, S., Energy, 30 (2005) 2370-2382.

(11) Merrill, M. J., and Z. Jin. Magnetic Resonance Imaging 12, 2: (1994) 345-348.

(12) Mansfield, P., and B. Issa. J. of Magnetic Resonance, A122, (1996) 137-148.

(13) Suekane, T., Yokouchi, Y., and Hirai, S., AIChE J., 41 (2003) 10-17.

(14) Callaghan, P. T., Principles of Nuclear Magnetic Resonance Microscopy. Clarendon Press, Oxford. (1991)

(15) Fukushima, E., Annu. Rev. Fluid Mech., 31, (1999) 95-123

(16) Bear, J., Dynamics of Fluids in Porous Media. Dover (1972) 441

(17) Kaviany, M., Principles of Heat Transfer in Porous Media Second Edition, Springer-Verlag New York Inc. (1995) 461-467 